\title{
Une génération de la Revue canadienne de l'enseignement des sciences, des mathématiques et de la technologie
}

\author{
Douglas McDougall
}

Accepted: 11 December 2020 / Published online: 15 January 2021

(C) Ontario Institute for Studies in Education (OISE) 2021

Très souvent, nous pensons qu'une génération dure environ 20 ans. La Revue canadienne de l'enseignement des sciences, des mathématiques et de la technologie (CJSMTE) a 20 ans. Dans ce numéro, nous poursuivons notre célébration qui a débuté dans le Volume 20 Numéro 3 avec Chernoff (2020) et Lajoie (2020). Dans le numéro 4 du volume 20, nous présentons les articles de nos auteurs qui ont accepté l'invitation à réfléchir au passé, au présent ou à l'avenir de la revue. Ces auteurs sont d'anciens éditeurs en chef, rédacteurs, membres du comité de rédaction et auteurs fréquents. Ce sont des sympathisants de longue date de la revue et je suis reconnaissant du temps qu'ils ont pris pour nous faire part de leur histoire et de leur éclairage alors que nous célébrons les 20 ans du CJSTME.

Nous commençons le numéro avec des articles des fondateurs. Derek Hodson (2021) écrit sur les questions clés qui doivent être abordées dans la construction d'un programme pour l'activisme sociopolitique. Il décrit un modèle en quatre étapes pour renforcer la capacité des étudiants à l'action sociopolitique sur les questions socioscientifiques (QSS). Il reconnait qu'il existe de nombreuses raisons pour lesquelles ce type de programme sera « extraordinairement complexe et difficile », mais en vaut la peine. Gila Hanna (2020a, b) commente, en anglais et en français, les 20 ans de la revue. Elle décrit les facteurs clés du succès continu de la revue, notamment un excellent comité de rédaction, la nomination de nouveaux rédacteurs et le fait qu'il ait réuni des chercheurs et des éducateurs anglophones et francophones. Elle pense que la revue peut « espérer avec confiance » une vie productive. Jacques Desautels (2021) donne un exemple de science et de politique dans L'enseignement des sciences et le politique : un exemple. Il se penche sur le programme révisé des sciences naturelles au niveau collégial au Québec et sur les parties prenantes qui ont contribué à la conception du programme. Cet article en français examine les enjeux politiques de la conception des programmes et comment Desautels revient là où il était il y a 20 ans.

Sous l'impulsion de ces trois auteurs et professeurs d'exception, John Wallace est devenu l'éditeur en chef de 2007 à 2018. Rina Zazkis et Nathalie Sinclair se sont jointes à lui au sein de l'équipe éditoriale. L'article de Zazkis (2021) portait sur les connaissances mathématiques dans l'enseignement en se fondant sur un numéro spécial intitulé « Personal Mathematical Knowledge in the Work of Teaching 》 (CJSMTE 13 (2), 2013). Elle intègre les idées présentées par les auteurs du numéro spécial 2013 à des exemples tirés de son propre enseignement. Sinclair (2021) se questionne quant à savoir si le moment est opportun pour

D. McDougall $(\bowtie)$

Ontario Institute for Studies in Education, University of Toronto, 252 Bloor Street West, Toronto, Ontario M5S 1V6, Canada e-mail: doug.mcdougall@utoronto.ca 
spatialiser, « transdiscipliner » et déconstruire le CJSMTE. Elle examine les articles du CJSTME des 20 dernières années pour examiner le rapport que le CJSMTE entretient avec les problèmes plus mondiaux de la recherche en éducation et comment les différents paradigmes de la recherche ont été représentés au fil du temps, en particulier pour l'enseignement des mathématiques.

Jérôme Proulx (2021) écrit sur diverses orientations en matière de didactique des mathématiques. Il écrit sur les dimensions communes et les distinctions pour clarifier le travail de recherche qui doit être fait tout en illustrant que le domaine est dynamique et en perpétuel renouvellement. Ann Kajander (2021) examine l'état de la recherche en mathématiques et comment nous pouvons progresser dans la compréhension de l'enseignement et de l'apprentissage des mathématiques. Elle revient sur des études antérieures et théorise sur le rôle de la recherche en enseignement des mathématiques à l'avenir.

Wolff-Michael Roth (2020) jette un regard en arrière et envisage l'avenir selon une optique personnelle pour fournir une perspective historique. Il utilise ses propres expériences en classe pour transformer les salles de classe et présente quelques idées de recherche futures qui pourraient faire l'objet d'études par les jeunes générations de chercheurs en SMTE. Cet axe de travail a été publié presque exclusivement dans le CJSMTE. Karen Goodnough (2021) réfléchit à l'apprentissage professionnel à travers le prisme d'une formatrice d'enseignants en sciences. Elle décrit comment la revue a contribué à son apprentissage professionnel grâce à ses divers rôles dans la revue : membre du comité de rédaction, auteure, critique et consommatrice. Elle donne aux formateurs d'enseignants en sciences de précieux conseils qui les aideront à atteindre leurs objectifs d'apprentissage professionnel.

Glen Aikenhead (2021) offre une vision des mathématiques et des sciences à l'école à l'aide de deux trames. La première histoire revient sur les articles portant sur l'amélioration et l'enrichissement de la science scolaire avec les modes de vie autochtones sur la Terre Mère. Une deuxième trame représente une perspective future sur l'amélioration des mathématiques scolaires avec la mathématisation autochtone, complétée par une étude récente qui fournit des détails sur cette nouvelle trajectoire. Erminia Pedretti et Ana Maria Navas Iannini (2021) se penchent sur l'évolution des objectifs et des rôles des musées scientifiques de quatrième génération. Elles envisagent que les musées scientifiques remodèlent leur identité pour promouvoir la citoyenneté active, la responsabilité sociale, l'engagement dans des questions scientifiques et technologiques complexes et l'agentivité. Elles suggèrent que ces institutions évoluent, se remettent en cause et se réorientent pour devenir davantage que ce qu'elles ont été historiquement.

Gary Pluim, Joanne Nazir et John Wallace (2021) explorent l'article de Basil Bernstein intitulé On the Classification and Framing of Educational Knowledge à travers le prisme de l'intégration des programmes. Les auteurs étudient plus particulièrement les bénéficiaires de l'intégration des programmes, en examinant six catégories. Ils proposent qu'une perspective mondiale constitue une approche qui nous ferait avancer dans les domaines des STEM. Auteur fréquent de la revue, Larry Bencze (2021) écrit sur l'enseignement activiste des sciences, des mathématiques et de la technologie en réexaminant les assemblages idéologiques. Il passe en revue les caractéristiques et les dommages importants liés aux sciences, aux mathématiques, à la technologie et à l'ingénierie, puis décrit un programme et un cadre pédagogique qui peuvent encourager et permettre aux étudiants d'imaginer et de promouvoir activement l'assemblage d'entités diverses en alliances qu'ils jugent appropriées.

David Blades (2021) a été le rédacteur fondateur de Newsround / Nouvelles brèves, auparavant une section du CJSMTE conçue pour «servir de moyen de rendre compte de l'actualité dans le domaine des SMTE à travers le pays et des personnes derrière ces événements » (Blades, 2001, p. 123). Larry Bencze, un autre auteur de ce numéro, a été le deuxième rédacteur en chef de Newsround / Nouvelles brèves jusqu'en 2007. Dans cet article, Blades démontre l'importance de favoriser un sentiment de communauté qui peut être atteint en publiant Newsround / Nouvelles brèves. Les rédacteurs du CJSMTE ont pris sa proposition comme un point de discussion important pour le volume 21 . 
Enfin, McDougall et Ferreyro Mazieres (2021) reviennent sur les 20 dernières années d'articles et de points de vue publiés dans le CJSMTE pour y déceler certaines tendances. Les résultats indiquent qu'il y a eu une augmentation des articles sur l'enseignement des mathématiques, équilibrant ainsi la proportion d'articles scientifiques par rapport aux articles mathématiques, ainsi qu'une concentration accrue sur les articles d'enseignement technologique et les articles sur les STEM au cours des cinq dernières années. Les auteurs proposent que l'équipe de direction de la revue envisage d'ajouter l'Afrique, en particulier des auteurs francophones, à l'appel à augmenter les voix internationales du CJSMTE au cours des prochaines années.

Le CJSMTE a été créé en 2001 pour bâtir une communauté d'éducateurs et de chercheurs en sciences, mathématiques et technologie, aux niveaux local, national et international. Les éditeurs en chef, les rédacteurs et les assistants de rédaction, de même qu'un comité de rédaction, des critiques et des auteurs qui le soutiennent, ont aidé la revue à étendre sa portée à l'échelle mondiale. Le journal est vieux d'une génération. Merci à chacun de vous pour le rôle que vous avez joué dans la construction de cette communauté. Ce numéro spécial nous a permis de célébrer 20 fabuleuses années et de nous lancer dans la troisième décennie du CJSMTE. On y va!

\section{Compliance with ethical standards}

Conflit D'intérêts Je ne déclare aucun conflit d'intérêts.

\section{Références}

Aikenhead, G. (2021). School Science and Mathematics Storylines. Canadian Journal for Science, Mathematics and Technology, 20(4). https://doi.org/10.1007/s42330-020-00115-5.

Bencze, J.L. (2021). Re-visioning Ideological Assemblages Through De-punctualizing and Activist Science, Mathematics \& Technology Education. Canadian Journal for Science, Mathematics and Technology, 20(4). https://doi.org/10.1007 /s42330-020-00133-3.

Blades, D. (2001). Newsround. Canadian Journal of Science, Mathematics and Technology Education, 1(1), 124-134.

Blades, D. (2021). Newsround: A Repetition. Canadian Journal for Science, Mathematics and Technology, 20(4). https://doi. org/10.1007/s42330-020-00122-6.

Chernoff, E.J. (2020). On the Occasion of an Anniversary, Eh: Confessions of a Canadian Math Ed Editor. Canadian Journal for Science, Mathematics and Technology, 20(3), 397-411.

Desautels, J. (2021). L'enseignement des sciences et le politique: un exemple. Canadian Journal for Science, Mathematics and Technology, 20(4). https://doi.org/10.1007/s42330-020-00131-5.

Goodnough, K. (2021). Professional learning and the Canadian Journal of Science, Mathematics and Technology Education: Reflections of a science teacher educator. Canadian Journal for Science, Mathematics and Technology, 20(4). https://doi. org/10.1007/s42330-020-00119-1.

Hanna, G. (2020a). In Celebration of the 20th Anniversary of the Canadian Journal of Science, Mathematics and Technology Education (CJSMTE). Canadian Journal for Science, Mathematics and Technology, 20(4). https://doi.org/10.1007 /s42330-020-00106-6.

Hanna, G. (2020b). À l'occasion du $20^{\mathrm{e}}$ anniversaire de la Revue canadienne de l'enseignement des sciences, des mathématiques et de la technologie (CJSMTE). Canadian Journal for Science, Mathematics and Technology, 20(4). https://doi.org/10.1007/s42330-020-00117-3.

Hodson, D. (2021). Going Beyond STS Education: Building a Curriculum for Sociopolitical Activism. Canadian Journal for Science, Mathematics and Technology, 20(4). https://doi.org/10.1007/s42330-020-00114-6.

Kajander, A. (2021). The mandate of scholarly mathematics education research: Moving ourselves forward. Canadian Journal for Science, Mathematics and Technology, 20(4). https://doi.org/10.1007/s42330-020-00121-7.

Lajoie, C. (2020). La Revue canadienne de l'enseignement des sciences, des mathématiques et des technologies: une vitrine de choix pour la diffusion en français de réflexions et recherches en didactique des mathématiques. Canadian Journal for Science, Mathematics and Technology, 20(3), 412-422.

McDougall, D. \& Ferreyro Mazieres, S. (2021). CJSMTE: 20 Years Strong. Canadian Journal for Science, Mathematics and Technology, 20(4). https://doi.org/10.1007/s42330-020-00120-8. 
Pedretti, E., \& Navas Iannini, A.M. (2021). A Towards fourth generation science museums: Changing goals, changing roles. Canadian Journal for Science, Mathematics and Technology, 20(4). https://doi.org/10.1007/s42330-020-00128-0.

Pluim, G., Nazir, J., \& Wallace, J. (2021). Curriculum Integration and the Semicentennial of Basil Bernstein's Classification and Framing of Educational Knowledge. Canadian Journal for Science, Mathematics and Technology, 20(4). https://doi. org/10.1007/s42330-021-00135-9.

Proulx, J. (2021). Des variétés de didactiques des mathématiques: réaffirmer le commun et tracer des distinctions. Canadian Journal for Science, Mathematics and Technology, 20(4). https://doi.org/10.1007/s42330-020-00129-z.

Roth, W-R. (2020). Looking Back and Looking Forward: A Historical Perspective on Science, Mathematics, and Technology Education in Canada Through a Personal Lens. Canadian Journal for Science, Mathematics and Technology, 20(4). https://doi.org/10.1007/s42330-020-00113-7.

Sinclair, N. (2021). Time to spatialise, transdiscipline and deconstruct CJSMTE? Canadian Journal for Science, Mathematics and Technology, 20(4). https://doi.org/10.1007/s42330-020-00127-1.

Zazkis, R. (2021). Personal, nonlocal, tacit: On mathematical knowledge in teaching. Canadian Journal for Science, Mathematics and Technology, 20(4). https://doi.org/10.1007/s42330-020-00118-2.

Publisher's Note Springer Nature remains neutral with regard to jurisdictional claims in published maps and institutional affiliations. 